\title{
Prevalence of Spondylolysis and Spondylolisthesis in Patients Afflicted with Chronic Back Pain in Babol City, Iran, during 2012 and 2013
}

\author{
Mahsa Layegh ${ }^{1}$, Ebrahim Hejazian ${ }^{2^{*}}$ \\ ${ }^{1}$ Medical Student, Babol University of Medical Sciences, Mazandaran, Iran \\ ${ }^{2} \mathrm{MD}$, Assistant Professor of Neurosurgery, Shahid Beheshti Hospital, Babol University of Medical Sciences, Mazandaran, \\ Iran \\ * Corresponding Author Address: Surgery and Neurosurgery Departments, Shahid Beheshti Hospital, Keshvari Square, \\ Shahid Sargord Ghasemi Street, Babol, Mazandaran, Iran. Tel:+981132252071. Fax:+981132251664. Postal Code: 8145147166. \\ Email: mersedeh_dadpour@yahoo.com \\ Article Type: Research Article \\ Received: April 12, 2017, Last Revised: May 23, 2017, Accepted: May 27, 2017, Published: June 30, 2017
}

\section{Abstract}

Background and Aim: Chronic back pain is one of the most important reasons of individuals' reference to clinic, so that no determined recognition is posed in considerable number of such individuals. Spondylolysis and spondylolisthesis are two important pathologies that people might be afflicted with for years but they might be unaware of it. Therefore, such diseases may account for chronic back pain. This study aims at analyzing prevalence of these two injuries in individuals afflicted with chronic back pain.

Methods and Materials/Patients: This has been a cross-sectional study for two years on individuals who referred to our clinic with complaining about chronic back pain with taken magnetic resonance imaging and radiography of spine for diagnosis of their problem. Information related to current pathologies in imaging was extracted and registered from an interpretation of physician and radiologist report.

Results: In this study, 289 out of 692 studied individuals were male. Spondylolysis and spondylolisthesis were observed in $8.6 \%$ and $13 \%$ of them, respectively. Prevalence of spondylolisthesis in women (18\%) was significantly more than that in men especially by aging. There was no statistically significant relationship between spondylolysis and spondylolisthesis.

Conclusion: Spondylolisthesis and spondylolysis account for chronic back pain in aged women with prevalence of $13 \%$ and $8.6 \%$, respectively.

Keywords: Chronic Back Pain; Spondylolysis; Spondylolisthesis; Prevalence

Please cite this paper as: Layegh M, Hejazian E. Prevalence of Spondylolysis and Spondylolisthesis in Patients Afflicted with Chronic Back Pain in Babol City, Iran, during 2012 and 2013. Iran J Neurosurg. 2017;3(1):8-14

\section{Introduction}

Chronic low back pain (LBP) is one of the most common chronic pains that people pay attention to [1]. Also, it accounts for most referring to clinics and hospitals [2]. Results of studies in the developed countries show that more than $85 \%$ of individuals experience back pain during their lifetime [3], that majority of them were treated without recognition analysis at the level of primary treatment services emergency cares and even in most people, this phenomenon was limited itself [4]. Since, principles for back pain do not suggest imaging techniques, and a lumbosacral simple graph will not be helpful in diagnosing the etiology of back pain $[5,6]$. But about one third of such individuals will be afflicted with chronic back pain that will entail a wide spectrum of inabilities during their lifetime [7]. No definite etiology for chronic LBP is specified in about two third of the individuals [7]. In Iran, there are small numbers of studies in this respect, and results of studies represent high break out of back pain in Iranian population and third grade of chronic LBP in creating load of this disease [8]. Therefore, more comprehensive studies in higher level of treatment services provide possibility of complex imaging studies such as magnetic resonance imaging (MRI) and multidirectional $\mathrm{X}$-ray (AP, lateral, oblique) to diagnose the etiology of chronic LBP in approach, 
treatment, and prognosis of such disease will be influential.

Spondylolysis (Figure 1) is a type of stress fracture in the pars interarticularis of lumbar spines that is discovered accidentally in spine imaging [9]. One of the main reasons of spondylolysis is irregular sport activities in the teenagers and adults that may result in pars interarticularis fracture. But as it has no specific sign, people might be afflicted for years [10]. As this injury in afflicted individuals is not well diagnosed, breakout and occurrence of this disease in the general population are estimated to be at a low level [10]. Accordingly, results of some studies in the recent years represent a relationship between chronic LBPs and spondylolysis [11]. Therefore, this injury in individuals afflicted with chronic LBP should be considered as a probable reason, and necessary diagnostic modalities should be performed.

Spondylolisthesis (Figure 2) is forward slippage of one vertebra on lower vertebrae occurring for several reasons [1]. Spondylolysis and other diseases like degenerative spondylolisthesis are more common in elderly patients [12]. Break out of such injury in aged individuals is more than that in other age groups, therefore, such injury may not be diagnosed in most individuals and young persons for not being diagnosed [12]. Spondylolisthesis is considered as one of the etiologies for chronic LBPs in aged individuals. For this reason, in the approach toward patient afflicted with chronic LBP, it should be considered and analyzed [13].

Spondylolisthesis has many types. In the most common classifying system, Wilts classification, spondylolisthesis is divided into six types based on etiology [14]: dysplastic, isthmic, degenerative, traumatic, pathologic, and iatrogenic.

According to the degree of upper vertebrae slipping, Meyerding classifying system divides spondylolisthesis into five grades as follows [5]:

Grade 1: $0-25 \%$

Grade 2: $26-50 \%$

Grade 3: $51-75 \%$

Grade 4: 76- 100\%

Grade 5: over 100\%

Of the other reasons of back pain, lumbarization and sacralization of lumbosacral spines can be referred to that generally called lumbosacral transitional vertebrae (Figure 3).

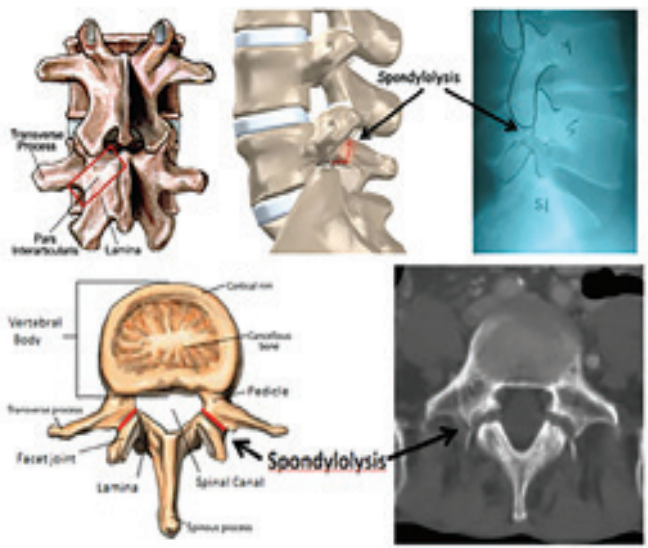

Figure 1. Spondylolysis, Defect in Pars Interarticularis

(Figure reproduced with permission: (c) 2017 RESURGENS ORTHOPAEDICS. Available at: http://jeffords.resurgens.com/spondylosis-lytic-spondylolisthesis.php)

Grades of spondylolisthesis

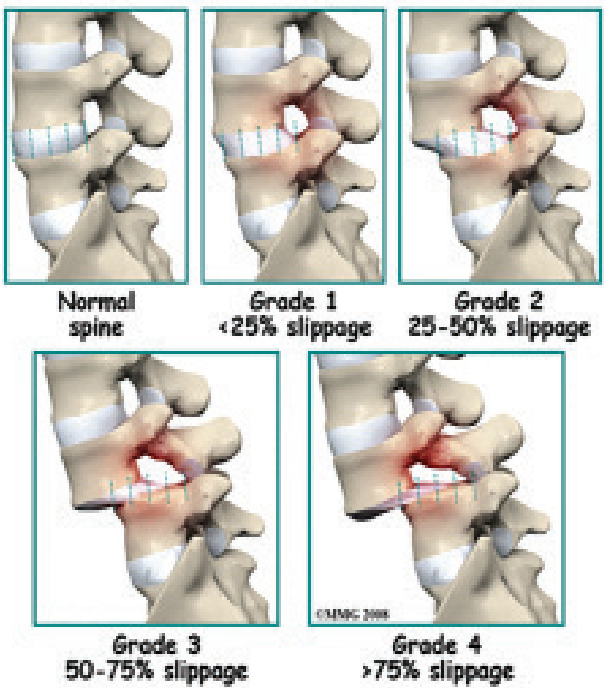

Figure 2. Grading of Spondylolisthesis

(Figure reproduced with permission: (c) 2017 Clinique du Dos Orthopole. Available at: http:// clinique-du-dos-bordeaux.fr/fra/les-pathologies/36-spondylolysthesis-degeneratif-et-par-lyse-isthmique) 


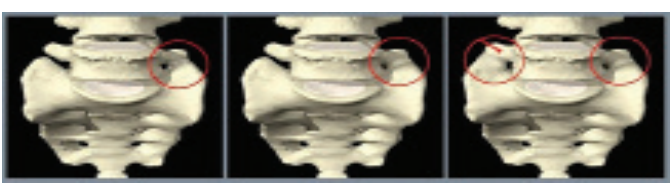

Figure 3. Sacralization

(Figure reproduced with permission: (C) 2015-2017 www.benhcoxuongkhop.net. Available at: http:// www.benhcoxuongkhop.net/benh-cung-hoa-dotsong-15-la-gi.html)

This state results from one congenital anomaly of lumber spine. The highest sacral segment and lowest lumbar segment are involved in lumbarization and sacralizasion, respectively (Figure 4). This happens in $4-40 \%$ of population [16].

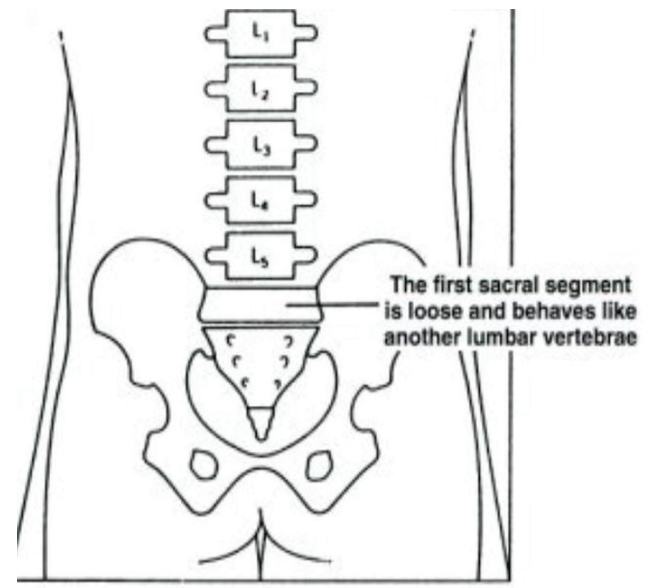

Figure 4. Lumbarization

(Figure reproduced with permission: (C) 2013 - 2016 SARAH KEY GLOBAL. Available at: http://www.simplebackpain.com/lumbarisation-and-sacralisation.html)

The precise etiology of chronic back pain in most of the individuals is not well recognized, and spondylolysis and spondylolisthesis may result in such signs. Few studies were administered in this respect, and there is a possibility to do studies with greater sample size especially in developing countries which have different lifestyles. In Iran, no study has been reported about this subject, yet. For this reason, this study aimed at analyzing the incidence of spondylolysis and spondylolisthesis in referring individuals with chronic LBP during 2012 and 2013.

\section{Methods and Materials/Patients}

In this cross-sectional study, all patients complaining about chronic LBP lasting more than three months during 2012 and 2013 who referred to our clinic were included. Lumbosacral spine MRI and X-ray imaging of lumber spine were taken. The referring patients with their imaging result were entered into the experiment, and demographic information of patients including age, sex, and data related to pain were registered in the information form. Findings related to MRI and X-rays were collected and registered in the information form using the radiologist's report and the interpretation of the neurosurgery specialist regarding the occurrence of spondylolisthesis, spondylolysis, sacralization, and lumbarization. Findings were analyzed using SPSS software (Version 21.0) and indexes of descriptive statistics, chi-square and independent sample t-test.

\section{Results}

During two years of study, 692 individuals afflicted with chronic LBP with lumbosacral spine MRI and X-rays imaging referring to clinics were entered into the study. Of all patients, 289 individuals (41.4\%) were male and 405 individuals were female. Mean age of patients was $41.9 \pm 14.1$. The maximum and minimum ages were 78 and 15 years, respectively. Mean age was $39.4 \pm 14.1$ and $43.6 \pm 12.7$ for men and women, respectively (p-value $<0.001$ ).

The occurrence of spondylolysis and spondylolisthesis in referring individuals were $60(8.6 \%)$ and $90(13 \%)$, respectively. Information of the most frequent sites of spondylolisthesis and spondylolysis are presented in the table 1 .

Radiologic findings related to prevalence of lumbosacral spine pathologies are depicted in figure 5 .

Results of analyzing the relationship between sex and LBP showed a significant relationship between female sex and spondylolisthesis (Table 2).

In analyzing the relationship between age and incidence of spondylolisthesis and spondylolysis in both sexes, age of women afflicted with spondylolisthesis was significantly more than that of healthy women. Age of individuals suffering from spondylolysis was significantly less than that of healthy ones in both men and women (Table 3).

On the relationship between occurrence 
Table 1. Prevalence and Location of Pathology

\begin{tabular}{|c|c|c|c|}
\hline Lesion & $\begin{array}{c}\text { Prevalence } \\
\text { (Percent) }\end{array}$ & Location of Lesion & Most Common Level \\
\hline \multirow{3}{*}{ Spondylolysis } & \multirow{3}{*}{$60(8.6 \%)$} & Right, (17) (28.3\%) & \multirow{3}{*}{ L5, (39) $(65 \%)$} \\
\hline & & Left, (25) (41.6\%) & \\
\hline & & Bilateral, (18) (30\%) & \\
\hline Spondylolisthesis & $90(13 \%)$ & ----- & L4-L5 (35) (38.8\%) \\
\hline
\end{tabular}

Table 2. Analyzing the Prevalence of Different Pathologies according to Sex

\begin{tabular}{|c|c|c|c|c|}
\hline Sex (N) & Spondylolysis (N) (\%) & $\begin{array}{c}\text { Spondylolisthesis } \\
(\mathbf{N})(\%)\end{array}$ & $\begin{array}{c}\text { Lumbarization } \\
(\mathbf{N})(\%)\end{array}$ & $\begin{array}{c}\text { Sacralization } \\
(\mathbf{N})(\%)\end{array}$ \\
\hline Male (289) & $12(7.6 \%)$ & $17(5.9 \%)$ & $10.3 \%)$ & $26(9 \%)$ \\
\hline Female (405) & $38(9.4 \%)$ & $73(18 \%)$ & 0.031 & $31(7.7 \%)$ \\
\hline p-value & 0.486 & $<0.001$ & 0.526 \\
\hline
\end{tabular}

Table 3. Relationship between Spondylolisthesis and Spondylolysis in Two Sexes

\begin{tabular}{|c|c|c|c|c|}
\hline Lesion & Index & Mean Age (years) & Deviation Value & p-value \\
\hline \multirow{4}{*}{ Spondylolisthesis } & Male, afflicted & 45.3 & 15.8 & 0.076 \\
\cline { 2 - 5 } & Male, normal & 39 & 14 & $<.076$ \\
\cline { 2 - 5 } & Female, afflicted & 50.5 & 12.1 & $<0.001$ \\
\cline { 2 - 5 } & Female, normal & 42.1 & 12.3 & $<0.001$ \\
\hline \multirow{5}{*}{ Spondylolysis } & Male, afflicted & 30.5 & 9.6 & $<0.001$ \\
\cline { 2 - 5 } & Male, normal & 40.1 & 14.2 & $<0.001$ \\
\cline { 2 - 5 } & Female, afflicted & 37.4 & 13.1 & 0.001 \\
\cline { 2 - 5 } & Female, normal & 44.3 & 12.5 & 0.001 \\
\hline
\end{tabular}

of synchronic spondylolisthesis and spondylolysis, it was found that only nine individuals $(10 \%$ of patients with spondylolisthesis) were afflicted with spondylolysis ( $p$-value $=0.626$ ), and most of the spondylolisthesis cases were caused by degeneration.

In an analysis on 90 patients with spondylolisthesis, 67 individuals (74.4\%) were afflicted with grade one, 22 individuals $(24.4 \%)$ with grade two and one person with grade five.

The most common level of involvement in spondylolisthesis among patients in this study was L4-L5, and the level of L2-L3 had the least common of involvement. Incidence of involved levels is shown in table 4.

Of 60 patients with spondylolysis, the most involved vertebra was L5 observed in 39 individuals (65\%). The L4 and L3 vertebrae were afflicted with spondylolysis in 17 $(28.3 \%)$ and $4(6.6 \%)$ patients, respectively.

Of all patients, 11 ones $(1.6 \%)$ had lumbarization, of whom one individual was male. 57 patients $(8.2 \%)$ had sacralization, of whom 26 individuals were male and 31 ones were female.

\section{Discussion}

Results of this study revealed that prevalence of spondylolysis and spondylolisthesis in population suffering from chronic LBP were 8.6 and $13 \%$, respectively. Moreover, spondylolisthesis prevalence in women was significantly higher han that in men. On investigating the relationship between age and various pathologies, women suffering from spondylolisthesis were found to be significantly older than men, and the individuals who suffered from spondylolysis 


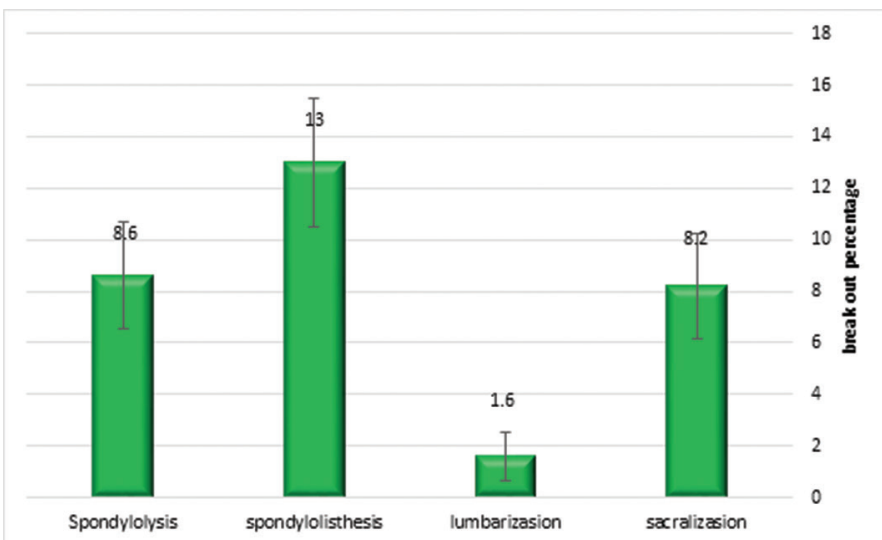

Figure 5. Prevalence of Different Pathologies Observed by X-ray Imaging in Patients Afflicted with Chronic Low Back Pain (694 Individuals) with $95 \%$ Confidence Interval

in both women and men were significantly younger.

Prevalence of spondylolysis in communities, ethnicities, age groups, and both sexes is different, and various studies have reported the prevalence as $5-20 \%(10)$. In our study, prevalence of spondylolysis is $8.6 \%$. Similar to most studies such as Kalichman et al. in 2009 (13) and Ko et al. in 2011(11), this study found no relationship between spondylolysis, as one of the basic factors, and back pain, while some of the studies like Niggemann et al. in 2011 (17) introduced spondylolysis as one of the main factors of chronic LBP, especially in patients with radicular pains. However in our study, radicular pain occurred in more than $60 \%$ of individuals, and the prevalence of spondylolysis was less common. This research realized that the prevalence of spondylolysis in both sexes were the same, while other studies $(10,11,13)$ reported that the prevalence of this injury in men was more than that in women. The authors of the present work concluded that individuals with spondylolysis were significantly younger than individuals without it, confirming the findings of most of the prior researches. In general, it is inferred that the prevalence of spondylolysis in people suffering from chronic LBP is low, and therefore it is not considered as main factor of LBP.

Spondylolisthesis can be one of the causes of chronic LBP, including two main etiologies: spondylolysis and degenerative spondylolisthesis. In this research, the prevalence of spondylolisthesis was $13 \%$ in patients suffering chronic LBP, however,
$18 \%$ in women with chronic LBP. More importantly, there was a statistically significant relationship between age and spondylolisthesis in women. Furthermore, no significant relationship existed between spondylolysis and spondylolisthesis, and only $10 \%$ of the individuals suffering from spondylolisthesis had spondylolysis. In a study performed by Frennered et al. in 1994 (18), the prevalence of spondylolisthesis in patients with LBP was estimated less than that in the findings of present study, while recent studies like He et al. (2014) estimated prevalence of spondylolisthesis higher than what was approved in our study (19). In most of the studies like the present one, women are more involved than men, and prevalence of spondylolisthesis is associated with increasing age in females (18-20). The most common etiology of spondylolisthesis is degeneration of joint surface, and spondylolysis is less important to make spondylolisthesis. So, it is supposed that its prevalence has particularly been increasing among elderly women within the recent years, and degenerative spondylolisthesis has mostly been responsible for chronic LBP in this group.

\section{Conclusion}

In general, the results of present study showed that prevalence of spondylolisthesis and spondylolysis were low in a selected population with chronic LBP in north of Iran. However, the prevalence of spondylolisthesis among women was significant and associated with increasing age. Therefore, spondylolisthesis is 
considered as one of the important causes of chronic LBP among old women. It is suggested that related clinical and paraclinical evaluations be performed in case of chronic LBP in aged women. Performing multi-center studies in other regions of the country with higher sample size as well as doing analytical studies, particularly cohort ones are recommended in order to more precisely determine the relationship between these injuries and prevalence of chronic LBP.

Table 4. Incidence of Involved Levels of Lumbosacral Vertebrae

\begin{tabular}{|c|c|c|}
\hline Level of Involvement & Number & Percent (\%) \\
\hline L2-L3 & 2 & 0.3 \\
\hline L3-L4 & 18 & 2.6 \\
\hline L4-L5 & 35 & 5 \\
\hline L5-S1 & 26 & 3.7 \\
\hline Multilevel & 9 & 1.3 \\
\hline
\end{tabular}

\section{Acknowledgement}

We appreciate Dr. Hamid Reza Mahdizadeh who contributed a lot in writing this article.

\section{Funding}

None

\section{Conflicts of Interest}

All authors of the study declare that they did not enjoy any potential or actual and material or spiritual benefits in doing the research.

\section{Authors' Contribution}

Conception and Design: Both authors

Data Collection: Both authors

Drafting the Article: Both authors

Critically Revising the Article: Both authors Reviewed Submitted Version of Manuscript: Both authors

\section{Approved the Final Version of the Manuscript: Both authors}

\section{References}

1. Stannard C, Kalso E, Ballantyne J. Evidence-Based Chronic Pain Management: Wiley; 2011.

2. Hoy D, Brooks P, Blyth F, Buchbinder R. The Epidemiology of low back pain. Best Pract. Res. Clin. Rheumatol. 2010;24(6):769-81.

3. Dunn KM, Hestbaek L, Cassidy JD. Low back pain across the life course. Best Pract. Res. Clin. Rheumatol. 2013;27(5):591-600.

4. Refshauge KM, Maher CG. Low back pain investigations and prognosis: a review. British journal of sports medicine.
2006;40(6):494-8.

5. Koes BW, van Tulder MW, Thomas S. Diagnosis and treatment of low back pain. Bmj. 2006;332(7555):1430-4.

6. Chou R, Deyo RA, Jarvik JG. Appropriate use of lumbar imaging for evaluation of low back pain. Radiol Clin North Am. 2012;50(4):569-85.

7. O'Sullivan P. Diagnosis and classification of chronic low back pain disorders: maladaptive movement and motor control impairments as underlying mechanism. Man Ther. 2005;10(4):242-55.

8. Mousavi SJ, Akbari ME, Mehdian H, Mobini B, Montazeri A, Akbarnia B, et al. Low back pain in Iran: a growing need to adapt and implement evidence-based practice in developing countries. Spine. 2011;36(10):E638-46.

9. Standaert CJ, Herring SA, Halpern B, King O. Spondylolysis. Phys Med Rehabil Clin N Am. 2000;11(4):785-803.

10. Sakai T, Sairyo K, Suzue N, Kosaka H, Yasui N. Incidence and etiology of lumbar spondylolysis: review of the literature. J Orthop Sci. 2010;15(3):281-8.

11. Ko SB, Lee SW. Prevalence of spondylolysis and its relationship with low Downloaded from back pain in selected population. Clin Orthop Surg. 2011;3(1):34-8.

12. Denard PJ, Holton KF, Miller J, Fink HA, Kado DM, Yoo JU, et al. Lumbar Spondylolisthesis Among Elderly Men. Spine. 2010:1

13. Kalichman L, Kim DH, Li L, Guermazi A, Berkin V, Hunter DJ. Spondylolysis and spondylolisthesis: prevalence and association with low back pain in the adult communitybased population. Spine. 2009;34(2):199-205.

14. Wiltse LL. Classification, Terminology and Measurements in Spondylolisthesis. Iowa Orthop J. 1981;1:52-57.

15. Niggemann P, Kuchta J, Grosskurth D, Beyer HK, Hoeffer J, Delank KS. Spondylolysis and isthmic spondylolisthesis: impact of vertebral hypoplasia on the use of the Meyerding classification. $\mathrm{Br} \mathrm{J}$ Radiol. 2012;85(1012):358-362. doi: $10.1259 / \mathrm{bjr} / 60355971$.

16. French HD, Somasundaram AJ, Schaefer NR, Laherty RW. Lumbosacral Transitional Vertebrae and Its Prevalence in the Australian Population. Global Spine J. 2014;4(4):229232. doi: $10.1055 / \mathrm{s}-0034-1387808$.

17. Niggemann P, Kuchta J, Beyer HK, Grosskurth D, Schulze T, Delank KS. Spondylolysis and spondylolisthesis: prevalence of different forms of instability and clinical implications. Spine. 2011;36(22):E1463-8.

18. Frennered K. Isthmic spondylolisthesis among patients receiving disability pension under the diagnosis of chronic low back pain syndromes. Spine.

1994;19(24):2766-9.

19. He LC, Wang YX, Gong JS, Griffith JF, Zeng XJ, Kwok AW, et al. Prevalence and risk factors of lumbar spondylolisthesis in elderly Chinese men and women. Eur Radiol. 2014;24(2):441-8

20. Virta L, Ronnemaa T. The association of mild-moderate isthmic lumbar spondylolisthesis and low back pain in middle-aged patients is weak and it only occurs in women. Spine. 1993;18(11):1496-503

\section{Comments}

This article present the results of a survey on prevalence of spondylolysis and spondylolisthesis in patients with chronic low back pain (LBP) in Babol. In this survey, 692 individuals were studied which makes it a unique work in this field and I could not find previous similar evaluation performed in Iran. In this study, the prevalence of spondylolysis and spondylolisthesis were $8.6 \%$ and $13 \%$, respectively, consistent with previous plain radiograph-based studies suggesting a prevalence in the adult population 
as $6 \%$. But the prevalence is about twice $(11.5 \%)$ in the CT-based studies. CT is the most accurate imaging modality for identification of spondylolysis and often reveals the presence of non-displaced spondylolysis when plain radiographs appear normal. If the authors have used CT for evaluation, the prevalence of spondylolysis would have become more than this.

Another issue worthy of attention is that the authors did not consider dynamic spondylolisthesis and did not use dynamic X-rays which is widely recognized as an effective method to detect the lumbar instability.

The level of spondylolisthesis was reported and the most common level (L4-L5) was consistent with previous studies in degenerative spondylolisthesis. Although the authors did not mention the prevalence of each type of spondylolisthesis separately, it seems that most of the patients had a degenerative type.

A challenging issue not fully addressed in this article is the relationship between spondylolysis, spondylolisthesis, and LBP. Is it necessary to perform X-ray evaluation in all patients with chronic LBP? The statistical analysis of the relationship between LBP and spondylolysis and spondylolisthesis was not presented but considering the low prevalence of these findings in LBP patients, it seems that the chronic pain may not be related and does not have good positive predicative value for spondylolysis and spondylolisthesis. Current study made no attempt to correlate radiographic findings with clinical symptoms which should be sought in future efforts.

In conclusion, to my knowledge, this is the first study that has assessed the prevalence of spondylolysis and spondylolisthesis in Iranian population. Although it has a few drawbacks, it can be a basis for additional research in this area.

Amir Azarhomayoun, $M D$

Department of Neurosurgery, Sina Hospital, Tehran University of Medical Sciences, Tehran, Iran

14 Iran J Neurosurg. 2017;3(1) 